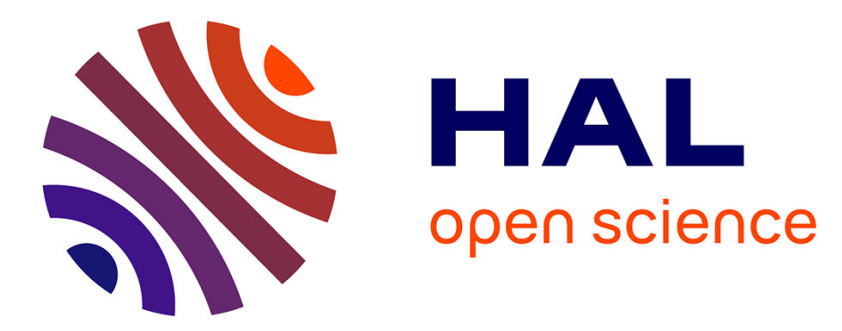

\title{
Operation of a low-power Hall thruster: comparison between magnetically unshielded and shielded configuration
}

Laurent Garrigues, S Santhosh, L. Grimaud, Stéphane Mazouffre

\section{To cite this version:}

Laurent Garrigues, S Santhosh, L. Grimaud, Stéphane Mazouffre. Operation of a low-power Hall thruster: comparison between magnetically unshielded and shielded configuration. Plasma Sources Science and Technology, 2019, 28 (3), pp.034003. 10.1088/1361-6595/ab080d . hal-02326255

\section{HAL Id: hal-02326255 \\ https://hal.science/hal-02326255}

Submitted on 23 Oct 2019

HAL is a multi-disciplinary open access archive for the deposit and dissemination of scientific research documents, whether they are published or not. The documents may come from teaching and research institutions in France or abroad, or from public or private research centers.
L'archive ouverte pluridisciplinaire HAL, est destinée au dépôt et à la diffusion de documents scientifiques de niveau recherche, publiés ou non, émanant des établissements d'enseignement et de recherche français ou étrangers, des laboratoires publics ou privés. 


\section{Operation of a Low-Power Hall Thruster: Comparison between}

\section{Magnetically Unshielded and Shielded Configuration}

L. Garrigues ${ }^{1, *}$ S. Santhosh ${ }^{1}$, L. Grimaud ${ }^{2}$, and S. Mazouffre ${ }^{2}$

${ }^{1}$ LAPLACE, Université de Toulouse, CNRS, 31062 Toulouse, France.

${ }^{2}$ ICARE, 1 C Avenue de la Recherche Scientifique, 45100 Orléans, France

The operation of a $250 \mathrm{~W}$ low-power Hall thruster called ISCT-200 has been studied using a two-dimensional hybrid model. Two different magnetic field topologies have been tested. One topology is called unshielded configuration and corresponds to a standard magnetic configuration with a quasi-radial magnetic field, and a second one, so called magnetic shielding where the zone of maximum of magnetic field is shifted in the near field plume. In that specific configuration, close to the channel walls, magnetic lines are forced to be parallel to walls. In the shielded configuration, the ionization takes place very close to the exhaust region and the acceleration occurs downstream the exit plane in the near field plume. The magnetic shielding configuration reduces the erosion very effectively since the cooling of the electron temperature inside the channel strongly diminishes the sheath potential drop and consequently the kinetic energy of ions impacting on channel walls. The shift of ionization and acceleration regions towards the near field plume also contributes to the reduction of erosion. Calculations show very similar performances for both magnetic field configurations, with a larger than measured thrust in the shielded version of the thruster. Also, thanks to a larger electron temperature a larger fraction of doubly charged ions is found in the shielded magnetic configuration of the ICST-200.

\footnotetext{
*Corresponding author; email: laurent.garrigues@laplace.univ-tlse.fr
} 


\section{Introduction}

The progress in electronics and the reduction of rocket launch cost over the last decade has driven the development of new spacecraft architectures. A few trends have emerged from these advancements. One is the greater use of electric propulsion systems for both stationkeeping and orbit raising. In this domain Hall thrusters (HT) are one of the most widespread electric propulsion technologies [1].

The other trend spurred by these changes is the emergence of small satellites. They take advantage of more modern miniaturized systems to perform the same tasks as previous generation spacecraft but in a smaller cheaper package. However these platforms often lack dedicated propulsion solutions as most of the efforts in the past decades have been focused on the 1 to $10 \mathrm{~kW}$ power range. For those applications, low power Hall thrusters can be particularly interesting. They combine high thrust to power ratio with moderate specific impulse and high efficiency which makes them ideally suited for near Earth operation. They also benefit from a long flight heritage further reducing development and qualification costs.

One of the main limitations of low power $(<500 \mathrm{~W})$ Hall thrusters is their lifetime. While thrusters in the 1 to $5 \mathrm{~kW}$ range can achieve up to 10000 hours of operation [2] small HT are usually limited to 3000 hours [3]. The life limiting factor for HT is the erosion of the discharge channel by fast ions. This sputtering problem is particularly acute in small thrusters where the surface to volume ratio is high [4].

A solution to this issue is the "magnetic shielding" (MS) configuration first proposed by the Jet Propulsion Laboratory [5]. This technique relies on a specific magnetic topology inside the thruster and reduces erosion by at least two orders of magnitude [6]. The MS configuration applied to small HTs could enable missions inconceivable today by dramatically increasing the total impulse provided by the propulsion system [7]. In this paper 
hybrid model simulations of two magnetic field configurations MS and "unshielded" (US) are reported and deeply analyzed for a $200 \mathrm{~W}$-class HT. The rest of the paper is organized as follows. In section II the fundamentals of MS configuration and thruster description in the US and MS configurations are presented. In section III the hybrid model is presented. Section IV is dedicated to the simulation results and analysis of both magnetic configurations. We summarize the main results and give some perspectives in section $\mathrm{V}$.

\section{Common notions about magnetic shielding}

In this section, we briefly come back on the general ideas about the unshielded and magnetic shielded versions of HTs. The presented trends will be revisited in section IV through calculations of the two magnetic configurations.

\section{A. Magnetic shielding topology}

In a standard unshielded Hall thruster (US-HT) the magnetic field is mostly radial. The maximum value of the radial magnetic field is close to the exit plane of the thruster. In this area the electric field increases [8] leading to electrons to become very energetic. Since electron mobility is high along the field lines, it means that electrons are in direct contact with the walls and thus create a very energetic sheath at this location. Typical electron temperatures of a few tens of $\mathrm{eV}$ can result in sheath potential drops of few tens of volts when strong electron emission from the ceramic walls occurs [9]. Since magnetic field lines are not purely equipotential, the electric potential lines form a concave length meaning that ions generated close to the walls are accelerated towards them [10], as we can see in figure 1. This results in a high flux of energetic ions colliding with the walls and causing a lot of sputtering.

The magnetic topology of a magnetic shielded Hall thruster (MS-HT) is illustrated in figure 1 . The main characteristic of the magnetic shielding is the presence of field line tangent to the wall reaching from the thruster's magnetic poles to the anode area. This "grazing line" 
produces a layer of cool electrons near the walls and thus reduces the sheath potential drop. In order to get this grazing line the maximum of the magnetic field needs to be pushed downstream of the exit plane of the thruster. In a MS-HT the acceleration region is mostly situated outside the thruster. Consequently, the electron temperature inside the channel is low and electric potential lines almost follow the magnetic field lines as illustrated figure 1 . The conical shape of the channel geometry is crucial [5], [6], [11], [12]. Those two combined effects have been experimentally observed [6] and contribute to reducing the erosion rate of the channel walls.

\section{B. Magnetic shielding and low power Hall thrusters}

Most of the efforts in developing magnetic shielding have been spent in high power thrusters. Notable examples include the $6 \mathrm{~kW} \mathrm{H6-MS} \mathrm{[13],} \mathrm{the} 20 \mathrm{~kW}$ NASA-300MS [14], the $12.5 \mathrm{~kW}$ HERMeS [15] and the $9 \mathrm{~kW} \mathrm{H9} \mathrm{[16].} \mathrm{For} \mathrm{all} \mathrm{those} \mathrm{thrusters} \mathrm{the} \mathrm{performances} \mathrm{are}$ on par with unshielded thruster of similar discharge power. In parallel with the experimental development of those thrusters, development of simulation tools capable of capturing the physics of the MS-HT discharge have been developed. In the US most of the attention has been focused on the Jet Propulsion Laboratory Hall2De code [6]. This code uses a 2D axissymmetric magnetic field aligned mesh. All charged particles are treated as a fluid. The atoms are considered non-collisional and their density is calculated with a line of sight algorithm. As with all codes using a fluid electron model an anomalous mobility is introduced to account for the anomalous transport of the electrons through the magnetic barrier. Combination of simulation results and experiments has permitted to assess the positive effect of the MS configuration on the limitation of wall erosion [17].

On the low power side, outside the work presented in this study, the main effort has been the MaSMi family of thrusters developed by Conversano [3]. The first thruster called the MaSMi-40 was designed for a discharge power around $300 \mathrm{~W}$ and achieved full magnetic 
shielding. An anode efficiency of 0.22 at a discharge power of $330 \mathrm{~W}$ has been obtained [18]. The larger MaSMi-60-LM1 was then developed and reached nearly 0.29 anode efficiency around $500 \mathrm{~W}$ [19]. This thruster was extensively simulated with Hall2De to understand why such a low efficiency is achieved [20]. Lessons learned from this study have led to the construction of a secondary laboratory model (LM2) [21] as well as a demonstration model (DM) increasing its performance to 0.45 anode efficiency [22].

\section{The ISCT-200 Hall thruster}

The reference thruster used in this study is the ISCT200 with two magnetic configurations un-shielded and magnetic shielding, respectively noticed ISCT200-US and ISCT200-MS in the rest of paper. The ISCT200 is a $200 \mathrm{~W}$ class, permanent magnet HT that has been manufactured at ICARE laboratory and tested in the NExET test chamber [8], [23]. We show in figure 2 the magnetic field strength profile along the thruster centerline for the two configurations. The maximum of the magnetic field strength is shifted outside the exit plane of the thruster, as expected. We also notice that both configurations exhibit a zero-B field in the middle of the channel, but the magnetic field profile in the anode region differs with a larger increase of B in the US configuration. No magnetic field optimization on that specific thruster has been performed. The ISCT-class HTs have been extensively characterized [8], [23], [24]. The axial ion velocity distribution in several key areas has been measured by LIF

spectroscopy [8]. While the normal discharge channel is made out of $\mathrm{BN}-\mathrm{SiO}_{2}$, testing with graphite discharge channel walls was performed [23]. In the MS case, the performance measured show a peak efficiency of around $24 \%$ at $250 \mathrm{~W}$ [25].

\section{Hybrid model description}

The hybrid model is two-dimensional (axial and radial directions are considered, namely $x$ and $r$, respectively), axisymmetric, starting from the anode plane at the rear of the channel and 
ending at the magnetic field line that intercepts the cathode (limit of neutralizing beam) for ions and electrons and at the external open boundary for neutrals. The channel end is conical. The magnetic field is preprocessed with the FEMM software [26] from the detailed knowledge of positions and properties of magnetic materials (permanent magnets and pole pieces). The self-magnetic field distribution induced by the plasma itself is neglected. A kinetic description is used to calculate the transport of heavy species, while the electron transport is represented with fluid equations, assuming a Maxwellian distribution function. Singly and doubly charged un-magnetized ions are considered. The model is quasineutral and sheaths are described analytically. The electron density is everywhere equal to ion density, and in the rest of the paper is referred to as plasma density. Typically, $100 \times 75$ computational cells are used in the calculations shown. A regular grid is used inside the channel region, while a sparse grid is employed in the near field region (see Figure 3).

\section{A. Kinetic description of heavy species}

As in Particle-In-Cell (PIC) simulation, the energy distribution of heavy species is sampled with a fixed number of macroparticle (or superparticle). At each time step heavy species trajectories are integrated according to Newton's law. In the discharge volume, new ions are generated according to the spatial profile of ionization source term, and neutral atoms are depleted accordingly. Ion species taken into account are singly (xenon ground state to first level of ionization of charge ) and doubly charged ions (from ground state to second level of ionization and the stepwise ionization from first level of ionization to second level of ionization). The rates are the same as in the study of Ref. [27]. Ions impinging the walls of the channel and the anode plane are neutralized and new neutrals return back in the computational domain, while ions passing the cathode line are eliminated. The thrust is calculated according to minus the electric force acting on the ions (the minor contribution of ions impacting on walls is also included). Neutrals are injected in the simulation domain through the injection 
region according to the mass flow. Neutrals colliding with walls are isotropically reflected according to a half Maxwellian distribution in the direction normal to the surface at a temperature of $\mathrm{T}_{\mathrm{w}}=500 \mathrm{~K}$ [28]. Neutrals crossing the open Cartesian boundary beyond the cathode line are eliminated. A supplementary injection of neutrals to account for the vacuum backpressure is considered.

Calculations of ion mean velocity at the wall reveal that the Bohm sheath criterion is not automatically satisfied since sheaths are not included in the quasineutral model. Parra and Ahedo [29] have shown that the choice in the grid spacing (fine or coarse) affects the gradient of ion density and velocity in the pre-sheath and consequently the ion mean velocity at the wall boundary. Even with a fine mesh, the capability of the quasineutral model to satisfy the Bohm sheath criterion is not achieved. A correction in the weighting scheme on the boundary nodes has been proposed to recover the attempts properties. We use another method proposed by Lampe et al. [30], extended by Ahedo et al. [31], forcing that the ion mean velocity normal to the wall being equal to the Bohm velocity, like a boundary condition. In the model, we define a strip $d_{w}$ at a small distance of the walls (typically $d_{w} \sim 150 \mu \mathrm{m}$, larger than $\mathrm{c}_{\mathrm{s}} \Delta \mathrm{t}-\mathrm{c}_{\mathrm{s}}$ being the sound speed, i.e. typically half the cell thickness adjacent to the wall) in which we calculate the Bohm velocity for singly and doubly charged ions. For the particles located in those strip, we add an increment of velocity in the component normal to the wall such that the ion mean velocity equals the Bohm velocity. In practice, after a few iterations, the presheath profile is established and the correction of velocity is later minor.

\section{B. Fluid electron transport}

The Hall parameter $H$, which is the ratio between the cyclotron frequency $\omega$ and the electron collisional frequency $v$, is on the order of $10^{3}$ in Hall thrusters meaning that electrons are strongly magnetized. We use a fluid collisional approach (three firsts moments of 
Boltzmann equation) to describe the electron transport, coupled with a quasineutral assumption. The plasma density $n$ is obtained from the calculations of ion densities:

$$
n=n_{i^{+}}+2 n_{i^{2+}}
$$

where $n_{i^{+}}$and $n_{i^{2+}}$ are respectively singly and doubly charged ion densities. The electric potential profile is no longer calculated from the Poisson's equation but from the coupling of electron momentum and continuity equations.

It is convenient to treat the electron transport in the two directions separately to construct a grid aligned on the magnetic field lines. The magnetic streamlines $\lambda$ are obtained from:

$$
\frac{\partial \lambda}{\partial x}=r B_{r}, \frac{\partial \lambda}{\partial r}=-r B_{x}
$$

$B_{r}$ and $B_{x}$ being the radial and axial coordinates of magnetic field. The $\lambda$ stream function is constant along the magnetic field lines $(\mathbf{B} . \nabla \lambda=0)$. The construction of the stream function requires a monotonic variation of $\lambda$. The US and MS configurations are specifics in the sense that a zero-B field ( $\mathrm{X}$ point) exists. We define 4 zones connected to each other through the $\mathrm{X}$ point (see Figure 3). The West, East, South, and North zones define the computational domain area corresponding to the location between the $\mathrm{X}$-point and the anode, cathode, inner and outer walls, respectively.

Along the magnetic field lines, an electron momentum equation under the drift-diffusion approximation in which the drift and diffusion terms are almost the same implies that the electric potential distribution can be determined from a Boltzmann's distribution. The electric potential is written under the form [32]:

$$
\emptyset(x, r)=\emptyset^{*}(\lambda)+\frac{2}{3 e} \varepsilon_{e}(\lambda) \ln \left[\frac{n(x, r)}{n_{0}}\right]
$$


where the electron mean energy is denoted as $\varepsilon_{e}, n_{0}$ is a reference density (constant). $\emptyset^{*}$ called the thermalized potential and $\varepsilon_{e}$ are functions depending on the $\lambda$ stream function. For Maxwellian electrons, $\varepsilon_{e}=\frac{3}{2} T_{e}$.

The electric potential profile is the result of the imposed potential drop between anode and cathode and electron conductivity perpendicular to the magnetic field (generalized Ohm's law). Across the magnetic field lines, the electron flux is $\Gamma_{e, \perp}$ written as:

$$
\Gamma_{e, \perp}=-\mu_{e, \perp}\left[n E_{\perp}+\frac{2}{3 e} \nabla_{\perp}\left(n \varepsilon_{e}\right)\right]
$$

where the index $\perp$ indicates the direction perpendicular to the magnetic field, $\mu_{e, \perp}$ and $E_{\perp}$ respectively are the cross field electron mobility and electric field, and $\nabla_{\perp}$ is the cross field gradient. To calculate the electric field profile, we substitute in Eq. (4) the current conservation equation:

$$
e \iint \Gamma_{e, \perp} d s=e \iint \Gamma_{i, \perp} d s-\beta I_{d}
$$

$\Gamma_{i, \perp}$ is the cross field ion flux, $e$ is the elementary charge, and integrals are taken along the magnetic field lines (surfaces). The discharge current $I_{d}$ is determined such that a given potential drop is applied between anode and cathode. The coefficient $\beta$ is equal to 1 in West and East zones that contain the anode and cathode, and $\beta$ is equal to 0 (perfect dielectric) in South and North zones where magnetic field lines connect the same wall.

We solve a one-dimensional energy equation perpendicular to magnetic field (integrated between two consecutive magnetic field lines) to determine the electron mean energy profile that involves in the calculations of electric potential and ionization source terms. The energy equation is written as:

$$
\frac{\partial\left(n \varepsilon_{e}\right)}{\partial t}+\frac{5}{3} \nabla_{\perp} \cdot\left(\Gamma_{e, \perp} \varepsilon_{e}\right)-\frac{10}{9 e} \nabla_{\perp} \cdot\left(\mu_{e, \perp} n \varepsilon_{e} \nabla_{\perp} \varepsilon_{e}\right)=-e E_{\perp} \cdot \Gamma_{e, \perp}-P^{c o l}-P^{W}
$$


$P^{c o l}$ and $P^{W}$ correspond to the energy losses due to inelastic collisions between electrons and heavy species and to electron-wall interactions inside the channel, respectively. $P^{c o l}$ is detailed in Ref. [27] and $P^{W}$ includes the secondary electron emission effect [33]:

$$
P^{W}=n W=n \frac{\iint_{S_{W}} \Gamma_{i, W} d s_{W}}{\iiint_{\mathrm{V}} n d v}\left\{\frac{4\left(\varepsilon_{e}-\bar{\delta} \varepsilon_{e, s}\right)}{3(1-\bar{\delta})}+\frac{2 \varepsilon_{e}}{3} \ln \left[(1-\bar{\delta}) \sqrt{\frac{m_{i}}{2 \pi m_{e}}}\right]\right\}
$$

where $\Gamma_{i, w}$ is the ion flux at the walls, $d s_{w}$ and $d v$ are wall surface and volume elements, and the surface and volume integrals are performed, respectively, on the wall surface $\left(s_{w}\right)$ and in the volume $(v)$ between two nearby magnetic field lines, $m_{i}$ and $m_{e}$ are the ion and electron mass, respectively.

In Eq. (7), the properties of the materials involves in $\bar{\delta}$ which is the effective total secondary electron emission yield after integration over a Maxwellian distribution function of the total secondary electron emission yield $\delta$ and in $\varepsilon_{e, s}$ related to mean energy of emitted electrons. All the properties taken in that study are the same as in Ref. [33].

In practice, in each zone, electron equations (3) to (6) are firstly solved between east and west zones. The potential is fixed in one point corresponding to the anode $(300 \mathrm{~V})$ and to cathode positions $(0 \mathrm{~V})$. At those positions, the electron mean energy is fixed to $2 \mathrm{eV}$. The solution of electric potential and mean energy found at the stream line delimiting the four zones are used as a boundary to solve the electron fluid equations in south and north zones with $\beta=0$ in Eq. (5) and assuming a null derivative of the electron temperature between the two last lines of north and south sub-domains. More numerical techniques about the electron model are detailed in Ref. [34]. 


\section{Erosion model}

The estimation of thruster wall erosion has been considered including in the model a calculation of sputtering processes under ion bombardment on the walls, the effect of neutrals is negligible since their energy is much smaller compared to that of ions (no fast neutrals are considered). The effect of re-deposition is neglected too. To determine the axial profile of erosion, we have discretized the channel walls in different elements of constant length (same as axial grid). For each axial element, we calculate the eroded thickness per unit time (erosion rate) $R_{w}$ that depends on the ion flux at the walls $\Gamma_{i, w}$, the properties of the wall materials (mass $M_{w}$ and mass density $\rho_{w}$ of the wall materials) and the sputtering yield Y (number of atoms ejected per incident ions):

$$
R_{w}=\frac{\Gamma_{i, w} M_{w}}{\mathcal{N}_{a} \rho_{w}}\left\langle Y\left(\varepsilon_{i, w}, \theta_{i, w}\right)\right\rangle
$$

In Eq. (8), $\mathcal{N}_{a}$ is the Avogadro's number. The brackets indicate an integration over all the ions impacting one panel of the wall. The sputtering yield $\mathrm{Y}$ is a complex function depending of the ion properties at the walls (incident energy and angle, respectively denoted as $\varepsilon_{i, w}$ and $\theta_{i, w}$ in Eq. (8)). We use the same method as in Ref. [35]. The sputtering energy threshold has been fixed to $30 \mathrm{eV}$.

We add a kinetic energy to the ions (of charge number $Z_{i}$ equal 1 and 2 for singly and doubly charged ions) and accelerated in the sheath corresponding to $Z_{i} e \emptyset_{s}$ that includes the secondary electron emission [36]:

$$
\phi_{s}=\frac{2 \varepsilon_{e}}{3 e} \ln \left[(1-\bar{\delta}) \sqrt{\frac{m_{i}}{2 \pi m_{e}}}\right]
$$




\section{Anomalous electron collision frequency}

The cross-field electron mobility involving in the momentum and energy equations (Eqs. (4) and (6)] incorporates the classical collisions and non-classical (anomalous) effects. We use an empirical electron mobility profile (or collision frequency) such that the calculated plasma properties correspond to experimental values. Through this strategy, obviously the understanding of the mechanism responsible for cross-field electron transport can not be captured. The electron mobility involving in Eqs. (4) and (6) is written as

$$
\mu_{e, \perp}=\frac{e}{m} \frac{\left(v_{c}+v_{a n}\right)}{\left(v_{c}+v_{a n}\right)^{2}+\omega^{2}}
$$

where $v_{c}$ is the electron collisional frequency that includes the contributions of electronneutral [37], and electron-ion collision frequencies [38], $v_{a n}$ is the fitted electron anomalous collision frequency, and $\omega$ is the cyclotron frequency.

In Refs. [20], [39], the constant in time anomalous collision frequency is fitted from measurements of electric potential and electron temperature along the thruster centerline. Jorns [40] is employing the technique of machine learning to be able from the $2 \mathrm{D}$ measurements of electric potential and electron temperature in the near field plume to derive an empirical formulation of the anomalous collision frequency and to identify the main contribution to anomalous transport. In this paper, we fit the anomalous collision profile $v_{a n}$ to match time-averaged measured and calculated ion velocity profiles as in Refs. [27], [41] (time integration had be done along few hundreds of microseconds, larger than the time for the breathing mode). The magnitude of the frequency is chosen to match the discharge current. We show in Figures 4 and 5 a comparison between measured and calculated ion velocity profiles and frequency profiles along the thruster centerline for a mass flow of 1 mg.s $\mathrm{s}^{-1}$ and a voltage of $300 \mathrm{~V}$ for the ICST200-US and ICST200-MS. 
The profiles of collision frequencies along the thruster centerline for the US version are given in Figure 4b. The electron-neutral and electron-ion collision frequencies are calculated while the anomalous frequency is fixed to match the ion velocity profile shown Fig. 4a. Not surprisingly, the minimum of anomalous frequency coincides with the position of the maximum of the magnetic field, very close to the exit plane, as already previously noticed (e.g. [10], [39]). Following Ref. [39], outside the channel, we have limited the anomalous frequency to the cyclotron frequency. This rather arbitrary condition maintains that the electrons are magnetized in the near field plume, that it is consistent with the hypothesis of the model (in that situation, the Hall parameter $H$ is equal to 1 ). The contribution of electronneutral collisions on the cross-field transport is important close to the anode where the gas is injected. The detail of anomalous frequency profile in that region is not important, as far as the electron-neutral collisions dominate. The electron-ion collisions play a negligible role on the cross-field transport. Same exercise has been done for the MS version, and as expected, since the acceleration takes place outside the channel (see Fig. 5a), the minimum of anomalous collision frequency is localized upstream the exit plane at the same location than the maximum of magnetic field strength (compare Figures 2 and 5b). Same conclusions about frequency profiles can be drawn with the MS configuration. The change of anomalous collision frequency from US to MS configuration can be summarized as a shift of profile coinciding with the axial shift of the zone of large magnetic field.

\section{Comparisons between unshielded and magnetic shielded configurations}

All the simulations have been carried out with a xenon mass flow of $1 \mathrm{mg} / \mathrm{s}$ and a discharge voltage of $300 \mathrm{~V}$. The backpressure is fixed to $0.5 \mathrm{mPa}$ (indicated if different). The geometry of the thruster is exactly the same, only the magnetic configuration changes. The 
time-averaged results are averaged over $1 \mathrm{~ms}$. We start the process by simulating the transport of neutrals without plasma. When the steady state regime is achieved, we use the neutral profile as an initiale profile for the plasma module (starting with a uniform plasma density of $10^{16} \mathrm{~m}^{-3}$ and an electron temperature of $2 \mathrm{eV}$ ).

\section{A. Plasma properties}

We show the time-averaged plasma properties of the US and MS configurations in figures 6 and 7, respectively. The axial and radial distances have been normalized to thruster channel length $L$ and outer radius $R$. A comparison of electric potential profiles of Fig. 6a and 7a clearly exhibits a shift of the acceleration region, associated with a shift of the maximum of magnetic field. In this US configuration, the acceleration region takes place from either side of the exhaust plane, while the acceleration region is concentrated in the near field plume in the MS configuration. In the MS case, a closer look to the axial variation of the electric potential inside the channel near the inner and outer walls shows a nearly flat potential profile between $302 \mathrm{~V} \pm 2.5 \mathrm{~V}$, as previously shown in the literature [20]. A set of near-wall probes along the inner and outer walls including the conical part in the MS configuration has been carried out to compare electric potential profiles in US and MS configurations but for a $6 \mathrm{~kW}$ class HT. In the MS configuration, a quasi-constant electric potential value (close to the discharge voltage) is obtained near the walls, while a drop of electric potential in the zone of strong magnetic field is observed in the US configuration [6]. In Figs 6a and 7a, the electric potential profile has a non-monotonic variation with a maximum on the channel centerline, close to the anode in the US configuration and close to the exhaust in the MS configuration. 2D Calculations of the MaSMi-60 operation reveal the same trend in the electric potential profile [20]. The maximum of potential drop is associated to the local electron temperature, few volts in the US configuration and $10 \mathrm{~V}$ in the MS configuration. Induced by gradient of plasma density, from that specific region, electrons reach the anode through diffusion (second 
term on the right-hand side of Eq. (4)). A qualitative comparison of electric potential lines and magnetic field lines agrees with scheme presented Figure 1. Inside the channel, the electric potential lens is concave in the US configuration and convex in the MS configuration.

Due to the shift of acceleration region, the electrons gain energy less deeply into the channel in the MS configuration, as shown in Figures $6 \mathrm{~b}$ and $7 \mathrm{~b}$. The maximum of electron temperature is larger in the MS configuration $53 \mathrm{eV}$, to be compared with $30 \mathrm{eV}$ in the US configuration. When $\bar{\delta}$ reaches a critical value $\overline{\delta_{c}}=1-8.3 \sqrt{m_{e} / m_{i}}$ [42] $\left(\overline{\delta_{c}} \approx 0.983\right.$ for xenon) the sheath becomes space charge saturated and the sheath potential drops to 1.2 time $\mathrm{T}_{\mathrm{e}}$. [see Eq. (9)]. Secondary electron emission in the space sheath saturation regime acts as a sink limiting the electron mean energy [see Eq. (7)]. Calculations show here that we are not in that specific regime (close to exhaust $\bar{\delta} \sim 0.8$ and 0.5 in the US and MS configuration, respectively). An increase of the discharge voltage would certainly lead to reach that specific regime for the US configuration, as noticed for kW-range HTs [33], [36], [43].

The maximum of $T_{e}$ is in the same range of 2D calculations of the MaSMi-60 [20] for a same voltage but a bit larger mass flow of xenon. In the MS configuration, a significant reduction of the electron temperature in the channel is visible $\left(\mathrm{T}_{\mathrm{e}} \leq 10 \mathrm{eV}\right)$, downstream the ionization region. The "grazing" line that connects the poles to the anode region leads to low energetic electrons coming from the anode region to be able to easily travel along the magnetic field lines, establishing a low electron temperature close to the walls. Near the inner and outer walls $\mathrm{T}_{\mathrm{e}}$ is almost constant $(2 \mathrm{eV} \pm 1 \mathrm{eV})$. The already presented set of near-wall probes has been used to compare electron temperatures in US and MS configurations for a 6 $\mathrm{kW}$-class HT. Experiments confirm that the electron temperature along the walls are between 2.5 and 3 times smaller in the MS configuration [6]. Unfortunately, same measurements have not been performed yet for low power MS HTs. One indirect confirmation of a lower electron 
temperature close to the walls in the MS configuration comes from the picture of the light emitted when both thrusters operate. While in the US configuration the light emission covers all the surface of the channel exhaust, a less intense zone exists close to the walls in the MS configuration [25]. This effect can be almost partially attributed to a lower electron temperature in the near-wall region in the MS case. Note that the maximum of electron temperature in the US case is consistent with the analytical fitting laws of Ref. [4] showing that the maximum of electron temperature can be expressed as $T_{e}^{\max }=a_{T} V_{d}$, with $a_{T}=0.12$ (typically $36 \mathrm{eV}$ for a voltage of $300 \mathrm{~V}$ ).

The ionization source terms including all the contributions to ionization processes are shown in Figures 6c and 7c. Axially, the ionization region starts from the region of zero-B field and stops at the end of the channel in the US and extends to the near-field plume in the MS configuration. The peak of ionization (almost the same for both configurations $\sim 10^{24} \mathrm{~m}^{-}$ $\left.{ }^{3} \mathrm{~s}^{-1}\right)$ is spatially shifted in the exhaust plane in the MS case. One difference is the total ion current produced inside the discharge (calculated by integration of the local source term and volume time the charge number and elementary charge), it reaches $1.07 \mathrm{~A}$ in the US configuration and increases to $1.54 \mathrm{~A}$ in the MS configuration. Radially, the ionization region covers the entire channel region while a zone of very low ionization is visible near the walls in the MS configuration. The explanation is directly linked to the electron temperature profile. In the US configuration, a second zone of ionization appears, associated to the increase of magnetic field upstream the region of zero-B field (see Fig. 2). Keep in mind that no magnetic optimization has been performed for that version of the ICST-200. Same trends have already been observed but for a $1.5 \mathrm{~kW}$-class HT [35]. The plot of ion flux vectors in Figs. 6c and 7c is very instructive. In both cases, we can easily delimitate a zone above which ions generated are directed towards the walls. This region depends on the ratio between radial and axial electric fields, since ions are not magnetized and created at almost zero energy. The 
magnitude of the ion current impacting on wall is linked to the local ionization source term. In the US case, the zone of ion losses corresponds to the anode region (since a maximum of electric potential exists) and to the peripheral zone of ion generation, where ions are accelerated towards the walls due to the concave shape of electric potential. In the MS case, the main reason of ion losses is the presence of the peak of electric potential at $309 \mathrm{~V}$ in the exhaust region. As we see in Fig. 7c, ions are accelerated towards the walls when they are generated on the left-side of the ionization source term and towards the exhaust over wise. We will come back on that specific point in the next section. The positive effect of the conical shape of channel geometry on the reduction of ion impacts on walls is also visible in Fig. 7c, since the ion flux vectors are almost parallel to the walls. Nevertheless, we must point that this reduction is concentrated on a small area and underlines that it contributes to enlarge the divergence. The influence of magnetic field configuration on the erosion will be examined in section IV.C.

The time variation of the discharge current for both magnetic configurations plotted in figure 8 presents very similar profiles. A peak at a frequency of $30 \mathrm{kHz}$ associated to the periodic depletion of neutrals is clearly visible. This oscillation called "breathing mode" or "predator-prey" in the literature in the $20-70 \mathrm{kHz}$ range signs most of HT operations and has been observed on US (e.g. [43-45]) and on MS (e.g. [16], [22]) configurations.

\section{B. Performance analysis}

To analyze and compare the performance of both configurations, we define a certain numbers of quantities associated to the efficiency. The thruster efficiency considering multiply charged ions has been derived in Refs. [19], [47]. Revisiting the derivation proposed in the literature when singly and doubly charged ions are considered, the anode efficiency $\eta_{a}$ can be separated in five efficiencies: 


$$
\eta_{a}=\frac{T^{2}}{2 \dot{m}_{a} P_{d}}=\eta_{m} \eta_{b} \eta_{V} \eta_{d} \eta_{c}
$$

where $T, \dot{m}_{a}, P_{d}$ are the thrust, the anode mass flow and electric power $\left(P_{d}=I_{d} V_{d}\right)$.

The mass (or propellant) utilization, beam current, beam voltage, and beam divergence efficiencies are given by:

$$
\eta_{m}=\frac{\dot{m}_{i}}{\dot{m}_{a}}, \eta_{b}=\frac{I_{i}}{I_{d}}, \eta_{V}=\frac{V_{b}}{V_{d}}, \eta_{d}=\cos ^{2} \theta
$$

where $\dot{m}_{i}$ is the ion mass flow rate, $I_{i}$ and $I_{d}$ are ion and discharge currents, $V_{b}$ and $V_{d}$ are beam and discharge voltages, $\theta$ can be related to the half-angle and the divergence defined as containing $90 \%$ [23] or $95 \%$ [24] of the collected ion current over a hemisphere facing the thruster. The mass utilization efficiency can be written as:

$$
\eta_{m}=\frac{m_{i} I_{d}}{e \dot{m}_{a}} \eta_{b} \sum_{i} \frac{\zeta_{i}}{Z_{i}}
$$

the last term is a correction factor to account for two ion species of current noticed $I_{i^{+}}$and $I_{i^{2+}}$ for singly and doubly charged ions, respectively:

$$
\sum_{i} \frac{\zeta_{i}}{Z_{i}}=\frac{I_{i^{+}+}(1 / 2) I_{i^{2+}}}{I_{i}}
$$

The last term on right-hand side of Eq. (11) is the charge efficiency $\eta_{c}$ :

$$
\eta_{c}=\frac{\left(\sum i \frac{\zeta_{i}}{\sqrt{Z_{i}}}\right)^{2}}{\sum_{i} \frac{\zeta_{i}}{Z_{i}}}
$$

where $\sum_{i} \frac{\zeta_{i}}{\sqrt{Z_{i}}}=\frac{I_{i^{+}+} \sqrt{(1 / 2)} I_{i^{2+}}}{I_{i}}$.

The calculations of ISCT-200 performance are summarized in Table 1. The backpressure corresponds to the PIVOINE-2G conditions. Measurements of performance and discharge 
currents reported here have been performed in the NExET facility [8], [23]. The discharge current oscillations (rms value) compare well with measurements in the US and MS configurations. The thrust has been measured in the PIVOINE-2G test bench [25]. The overall trends in performances in the US configuration are reproduced while disagreements for the MS configuration are evident. (The anode efficiency disagreement is linked to the difference in the thrust). A mass utilization efficiency $\eta_{m}$ of between 0.76 and 0.78 can be achieved in calculations and in the same range in experiments. (Same calculations without correction of doubly charged ions as measured, show a slight correction with $\eta_{m}$ of 0.74 and 0.76). Since we report measurements for two different facilities with a difference of backpressure of 10 between NExET and PIVOINE-2G facilities and to discriminate this effect, we have also performed calculations with a backpressure of $5 \mathrm{mPa}$. Changes are modest, increasing the backpressure induces higher mass utilization efficiency by 5 to $10 \%$ and others performances in the same range and cannot explain the difference in the MS configuration about the thrust.

We have also calculated the ion energy current distribution of all ions leaving the computational domain. As in experiments, we have calculated the half-angle containing $90 \%$ of the current, we obtain half-angles of 35 and 45 degrees for US and MS configurations (as expected from results of Table 1) to be compared with the quasi the same half-angles of 57 degrees measured at $0.6 \mathrm{~m}$ from exit plane in the NExET facility [23]. Certainly that charge exchange collisions in the plume affect the angular ion distribution increasing the ion population of the tail of the distributions at large angle, and, as a result, increasing the thruster divergence. Other effect in the far field plume not considered in the model can also modify the thruster divergence [46]. Nevertheless, the $50 \%$ difference between measured and computed thrust cannot solely be explained by the beam divergence difference (and facility effects). One possible explanation could come from the acceleration of ions. Coming back to figures 4 and 5, we can calculate from the ion velocity the maximum of energy gained by the 
singly charged ions, we obtain $250 \mathrm{eV}$ and $230 \mathrm{eV}$ for un-shield and shielded version of the ISCT-200, respectively. It leads to an estimation of the beam voltage efficiency of 0.83 and 0.76, while calculations give 0.83 and 0.9 . The difference between measurements and calculated data for the MS case are clear and can explain almost partly the difference in thrust level. The origin of that discrepancy about the beam voltage efficiency is actually not identified (cathode sheath drop, acceleration further in the plume, etc.). We want also to emphasize that the electron-wall interactions that are not considered on front planes can maybe also play a role since the electron temperature is higher in the near field region.

If we compare with others available data of the literature as the MaSMI-60-LM1 [19], for a discharge voltage of $300 \mathrm{~V}$ and an electric power comparable to our study (243 W), a thrust of $12 \mathrm{mN}$, an anode efficiency of 0.28 are obtained. The detail of terms involving in the anode efficiency of Eq. (11) shows a high beam voltage efficiency $\sim 0.9$, a beam current and a mass utilization efficiency efficiencies $\sim 0.6$, a beam divergence efficiency reduced to 0.7 . Measurements of divergence have been realized at $0.5 \mathrm{~m}$ from thrust exit. Same kind of experimental campaigns on a $6 \mathrm{~kW}$-class MS (H6MS) thruster have shown higher mass utilization (0.93) and beam current (0.87) efficiencies, about the same we can expect from same power-class US HT [17]. Computational studies focused on the ionization layer show that a shorter channel length for low-power MS HTs also reduces the length of the ionization layer explaining the reduction of the mass utilization efficiency [20]. In comparisons with kW-class HT, the mass utilization efficiency in the ISCT-200 US and MS cases indicates that the neutral flux is largely un-ionized, explaining somehow the low degree of the amplitude of current oscillations of figure 8. Focusing on the ISCT-200 MS, the region of ionization is concentrated in the center of the channel and pushed in the exhaust region in the zone with a conical shape of the thruster channel. In that region the electron temperature is low and the neutrals are less confined by the walls, leading to a possible decrease in the ionization 
efficiency. In such small thrusters, another important feature of the ionization is the enlarged contribution of the neutrals that are formed after neutralization of ions impacting on walls and that are re-ionized further in the channel. The cumulative ion current losses on the walls (including the contribution of losses on the anode plane), represent a contribution even larger than the ion current extracted for the MS case (see Table 1). Coming back to figures 6 and 7, the respective position of maximum of ionization source term and electric potential profiles lead to a large contribution of ions produced to be directed towards the walls and neutralized. This is a possible indirect explanation of the measured decrease in the wall and anode temperatures of only between 30 to 60 Celsius degrees passing from the US to the MS configuration [24].

Additional information in Ref. [17] concerns the fraction of multiply charged ions. ExB probes have been used to measure the spectra of ion energy distributions. According to the spectra, Gaussian fittings centered at different bias potentials are used to calculate the population of the different ion species. In the MaSMI-60 thruster, for a power of $250 \mathrm{~W}$, multiply charged ions represents around $30 \%$ of the total current $\left(\mathrm{Xe}^{2+}\right.$ and $\mathrm{Xe}^{3+}$ current fractions reaches $20 \%$ and $8 \%$, respectively). The contribution of multiply charged ions over the total current even represents $45 \%$ in H6MS [17]. Compared to the US configuration, and as noticed in the literature and in our calculations, more multiply charged ions are extracted from the thruster. This is an even indirect evidence of a high electron temperature in the MS case.

\section{Erosion estimation}

In figure 9 we compare the kinetic energy of the ions impacting on the walls and estimate the eroded thickness for 1000 hours of thruster operation for the two magnetic configurations. In Fig. 9a, in the US case, an increase of the mean kinetic energy of the ions impinging the walls is visible at $\mathrm{x} / \mathrm{L} \geq 0.6$. The sheath potential drop in the region of strong electron 
temperature can reach $80 \mathrm{~V}$ (value taken at a position $\mathrm{x} / \mathrm{L}=0.9$ where $\bar{\delta} \sim 0.83$ in agreement with theoretical value of Eq. (9)). The sheath potential is still large since we are not in the space charge sheath saturation regime. In the MS case, the sheath potential drop remains low and the mean energy stays almost independent of axial position and below $20 \mathrm{eV}$.

In figure $9 \mathrm{~b}$, the eroded thickness shown are only for a sputtering threshold of $30 \mathrm{eV}$ (a larger value of threshold induces a thickness close to zero in the MS configuration). The positive effect of the conical shape of the walls close to the exhaust is visible with a reduction of the eroded thickness in the US case. In agreement with results of Figure 6, we notice a slight increase of eroded thickness downstream the zero-B position due to ions generated in that region and whose energy gained in the radial direction is higher than the sputtering threshold. Beyond a quantitative calculation of the wall erosion, the effectiveness of the MS configuration on the erosion of the walls is obvious.

\section{Conclusions and perspectives}

We have modeled the operation of the ISCT-200 low-power Hall thruster whose channel geometry is chamfered at the end. Two magnetic field configurations have been tested. The first one corresponds to a standard topology with a zone of maximum of magnetic field located around the exhaust region of the channel (US - unshielded configuration). The second configuration has one with a zone of maximum of magnetic field displaced in the near field plume and the magnetic field lines are almost parallel to the walls connecting the anode region to the outer/inner exterior poles (MS - magnetic shielding configuration). The anomalous electron mobility profile has been fitted with the experimental measurements of the axial ion velocity at the channel centerline. The calculations have been performed for fixed discharge voltage $(300 \mathrm{~V})$ and mass flow $(1 \mathrm{mg} / \mathrm{s})$, for a discharge power of about 250 W. 
Calculation results have permitted to revisit the general trends reported in the literature confirming a quasi-negligible erosion of channel walls, a large production of doubly charged ions and very similar oscillation regimes of the discharge currents. From the deeper analysis of time-averaged plasma properties, we have seen that the MS configuration leads to a simultaneous push of the ionization region towards the exhaust of the thruster and the acceleration region towards the near field plume. Inside the channel, the low potential drop induces a low electron temperature and magnetic field lines to be almost equipotential (compared to the unshielded configuration). In the US configuration, the ionization extends all over the radial direction, while, in the MS case, the ionization region is concentrated to the center of the channel. In the US case, the high $\mathrm{T}_{\mathrm{e}}$ is responsible for a large radial electric field and a large sheath potential drop inducing a high erosion rate. On the other hand, in the MS configuration, the specific positions of ionization source term and electric potential profiles lead to a high ion current impacting on walls. The advantage of the MS configuration is the relatively low radial electric field and electron temperature when they bombard the walls maintaining a low kinetic energy of ions close to or under the erosion threshold.

Comparisons with measurements have shown similar thruster performance for the US case but an overestimation of the thrust in the MS version of the ISCT-200 that has been attributed to ion beam properties (larger acceleration of the ions and a lower beam divergence in the calculations). It could be advantageous to use the LIF diagnostic in two-dimensions to map both axial and radial components of ion velocity in the near field plume and to determine if the shifted ionization and acceleration regions is responsible for a larger beam divergence in MS configuration. Measurements of electron temperature are necessary to validate the very high calculated electron temperature in the MS configuration. The incoherent Thomson scattering diagnostic previously used in the context of fusion plasmas has been recently successfully used to measure electron properties on a cathode plasma source with a high 
sensitivity [48]. Such non-intrusive diagnostic is now planned to be used in HT discharges. The deviation of a constant electron temperature along magnetic field lines could also be checked. Calculations for different mass flow rates and discharge voltages will be performed and a possible transition to the space charge saturation regime will be examined. We finally intend to modify the magnetic field configuration in the shielded version of the ISCT-200 and its consequence on plasma and performance properties.

\section{Acknowledgments}

This work has been performed with the support of CNES, through the Research and Technology Program. L. Grimaud benefits from a CNES and Région Centre PhD grant. 


\section{References}

[1] S. Mazouffre, Plasma Sources Science and Technology 25, 033002 (2016).

[2] F. Marchandise, N. Cornu, F. Darnon, and D. Estublier, "PPS®1350-G Qualification Status 10500 h," 30 International Electric Propulsion Conference, Florence, Italy, paper IEPC-2007-164 (2007).

[3] R. Conversano, "Low-Power Magnetically Shielded Hall Thrusters," PhD thesis, University of California, Los Angeles, 2015.

[4] K. Dannenmeyer and S. Mazouffre, Journal of Propulsion and Power 27, 236 (2011).

[5] I. G. Mikellides, I. Katz, R. R. Hofer, D. M. Goebel, K. de Grys, and A. Mathers, Physics of Plasmas 18, 033501 (2011).

[6] R. R. Hofer, D. M. Goebel, I. G. Mikellides, and I. Katz, Journal of Applied Physics 115, 043303 (2014).

[7] I. Levchenko, K. Bazaka, Y. Ding, Y. Raitses, S. Mazouffre, T. Henning, P. J. Klar, S. Shinohara, J. Schein, L. Garrigues, M. Kim, D. Lev, F. Taccogna, R. W. Boswell, C. Charles, H. Koizumi, S. Yan, C. Scharlemann, M. Keidar, and S. Xu, Appl. Phys. Rev. 5, 011104 (2018).

[8] L. Grimaud and S. Mazouffre, Plasma Sources Science and Technology 26, 055020 (2017).

[9] E. Ahdeo, Physics of Plasmas 9, 4340 (2002).

[10] J. Bareilles, G. J. M. Hagelaar, L. Garrigues, C. Boniface, J. P. Boeuf, and N. Gascon, Physics of Plasmas 11, 3035 (2004). 
[11] I. G. Mikellides, I. Katz, and R. R. Hofer, "Design of a Laboratory Hall Thruster with Magnetically Shielded Walls, Phase I: Numerical Simulations", $47^{\text {th }}$ AIAA Joint Propulsion Conference, San Diego, CA, 2011, paper AIAA-2011-5809.

[12] D. Pérez-Grande, P. Fajardo, and A. Ahedo, "Evaluation of Erosion Reduction Mechanisms in Hall Effect Thrusters" $34^{\text {th }}$ International Electric Propulsion Conference, Kobe, Japan, paper IEPC-2015-280.

[13] R. R. Hofer, D. M. Goebel, I. G. Mikellides, and I. Katz, Journal of Applied Physics 115, 043304 (2014).

[14] H. Kamhawi, W. Huang, T. W. Haag, R. Shastry, G. C. Soulas, T. Smith, I. G. Mikellides, and R. R. Hofer, "Performance and Thermal Characterization of the NASA300MS 20 kW Hall Effect Thruster," $33^{\text {rd }}$ International Electric Propulsion Conference, Washington, DC, 2013, paper IEPC-2013-444.

[15] H. Kamhawi, W. Huang, T. Haag, J. Yim, L. Chang, L. Clayman, D. A. Herman, R. Shastry, R. Thomas, C. Griffith, J. Myers, G. Williams, I. G. Mikellides, R. R. Hofer, J. E. Polk, and D. Goebel, "Overview of the Development of the Solar Electric Propulsion Technology Demonstration Mission 12.5-kW Hall Thruster," 50 ${ }^{\text {th }}$ AIAA Joint Propulsion Conference, Cleveland, OH, 2014, paper AIAA-2014-3898.

[16] R. R. Hofer, S. E. Cusson, R. B. Lobbia, and A. D. Gallimore, “The H9 Magnetically Shielded Hall Thruster," $35^{\text {th }}$ International Electric Propulsion Conference, Atlanta, GE, 2017, paper IEPC-2017-232.

[17] I. G. Mikellides, R. R. Hofer, I. Katz, and D. M. Goebel, Journal of Applied Physics 116, 053302 (2014). 
[18] R. W. Conversano, D. M. Goebel, I. G. Mikellides, R. R. Hofer, T. S. Matlock, and R. E. Wirz, "Magnetically Shielded Miniature Hall Thruster: Performance Assessment and Status Update," 50 $0^{\text {th }}$ AIAA Joint Propulsion Conference, Cleveland, OH, 2014, paper AIAA-20143896.

[19] R. W. Conversano, D. M. Goebel, R. R. Hofer, I. G. Mikellides, and R. E. Wirz, Journal of Propulsion and Power 33, 975 (2017).

[20] R. W. Conversano, D. M. Goebel, I. G. Mikellides, R. R. Hofer, and R. E. Wirz, Journal of Propulsion and Power 33, 992 (2017).

[21] R. W. Conversano, D. M. Goebel, R. R. Hofer, and N. Arora, "Performance Enhancement of a Long-life, Low-power Hall Thruster for Deep-space Smallsats," IEEE Aerospace Conference, Big Sky, MT, 2017.

[22] R. W. Conversano, R. B. Lobbia, K. C. Tilley, D. M. Goebel, S. W. Reilly, I. G. Mikellides, and R. R. Hofer, "Development and Initial Performance Testing of a Low-Power Magnetically Shielded Hall Thruster with an Internally-Mounted Hollow Cathode," 35th International Electric Propulsion Conference, Atlanta, GE, 2017, paper IEPC-2017-64.

[23] L. Grimaud and S. Mazouffre, Journal of Applied Physics 122, 033305 (2017).

[24] S. Mazouffre and L. Grimaud, IEEE Transactions on Plasma Science 46, 330 (2018).

[25] L. Grimaud, S. Mazouffre, and C. Boniface, "Performance Comparison Between

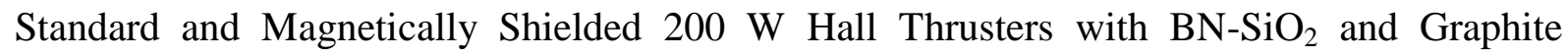
Channel Walls," $35^{\text {th }}$ International Electric Propulsion Conference, Atlanta, GE, 2017, paper IEPC-2017-172. 
[26] Finite Element Method Magnetics software, version 4.2, by D. Meeker, 2016 (URL: http://www.femm.info/wiki/HomePage).

[27] L. Garrigues, Journal of Applied Physics 119, 163305 (2016).

[28] L. Garrigues, G. Fubiani, and J. P. Boeuf, Journal of Applied Physics 120, 213303 (2016).

[29] F. I. Parra and E. Ahedo, "Fulfillment of the Bohm Condition on the 'HP Hall' FluidPIC Code", $40^{\text {th }}$ AIAA Joint Propulsion Conference, Fort-Lauderdale, FL, 2004, paper AIAA 2004-3955.

[30] M. Lampe, G. Joyce, W. M. Manheimer, and S. P. Slinker, IEEE Transactions on Plasma Science 26, 1592 (1998).

[31] E. Ahedo, R. Santos, and F. I. Parra, Physics of Plasmas 17, 073507 (2010).

[32] A. I. Morozov, Y. Esipchuk, G. Tilinin, A. Trofimov, Y. Sharov, and G. Y. Shchepkin, Soviet Physics Technical Physics 17, 38 (1972).

[33] L. Garrigues, G. J. M. Hagelaar, C. Boniface, and J. P. Boeuf, Journal of Applied Physics 100, 123301 (2006).

[34] G. J. M. Hagelaar, J. Bareilles, L. Garrigues, and J. P. Boeuf, Journal of Applied Physics 91, 5592 (2002).

[35] L. Garrigues, G. J. M. Hagelaar, J. Bareilles, C. Boniface, and J. P. Boeuf, Physics of Plasmas 10, 4886 (2003).

[36] S. Barral, K. Makowski, Z. Peradzynski, N. Gascon, and M. Dudeck, Physics of Plasmas 10, 4137 (2003). 
[37] J. P. Boeuf and L. Garrigues, Physics of Plasmas 25, 061204 (2018).

[38] N. Oudini, G. J. M. Hagelaar, J. P. Boeuf, and L. Garrigues, Journal of Applied Physics 109, 073310 (2011).

[39] I. G. Mikellides, A. L. Ortega, I. Katz, and B. A. Jorns, "Hall2De Simulations with a First Principles Electron Transport Model Based on the Electron Cyclotron Drift Instability," $52^{\text {nd }}$ AIAA Joint Propulsion Conference, Salt-Lake City, UT, 2016, paper AIAA-2016-4618.

[40] B. A. Jorns, "Data Driven Model for Electron Transport in a Hall Effect Thruster", Space Propulsion Conference, Seville, Spain, 2018, paper SP-277.

[41] L. Garrigues, S. Mazouffre, and G. Bourgeois, Journal of Applied Physics 111, 113301 (2012).

[42] G. D. Hobbs and J. A. Wesson, Plasma Physics 9, 85 (1967).

[43] N. Gascon, M. Dudeck, and S. Barral, Physics of Plasmas 10, 4123 (2003).

[44] T. Ito, N. Gascon, W. S. Crawford, and M. A. Cappelli, Journal of Propulsion and Power 23, 1068 (2007).

[45] R. R. Hofer, R. S. Jankovski, and A. D. Gallimore, Journal of Propulsion and Power 22, 721 (2006)

[46] I. G. Mikellides and I. Katz, Physical Review E 86, 046703 (2012).

[47] R. R. Hofer and A. D. Gallimore, Journal of Propulsion and Power 22, 732 (2006).

[48] B. Vincent, S. Tsikata, S. Mazouffre, T. Minea, and J. Fils, Plasma Sources Science and Technology 27, 055002 (2018). 
Table 1: Performance of ISCT-200 US and ISCT-200 MS. Experiments are taken from Refs. [8], [23], [25]. ${ }^{a}$ No correction for the presence of multiply charged ions has been applied, ${ }^{b}$ Estimated from figures 4 and 5.

\begin{tabular}{|c|c|c|c|c|}
\hline \multirow[b]{2}{*}{ Performance } & \multicolumn{2}{|c|}{ ISCT-200 US } & \multicolumn{2}{|c|}{ ISCT-200 MS } \\
\hline & Cal. & Exp. & Cal. & Exp. \\
\hline Discharge voltage $V_{d}(V)$ & 300 & 300 & 300 & 300 \\
\hline Anode mass flow $\dot{m}_{a}(\mathrm{mg} / \mathrm{s})$ & 1 & 1 & 1 & 1 \\
\hline Discharge current $I_{d}(\mathrm{~A})$ & 0.83 & 0.82 & 0.82 & 0.83 \\
\hline Discharge current oscillations $I_{d, o s c}(\mathrm{~A})$ & 0.07 & $<0.1$ & 0.09 & $<0.1$ \\
\hline Ion current $I_{i}(\mathrm{~A})$ & 0.59 & 0.52 & 0.63 & 0.52 \\
\hline Ion current losses $I_{i, w}(\mathrm{~A})$ & 0.48 & N/A & 0.91 & N/A \\
\hline Thrust $T(\mathrm{mN})$ & 14.1 & 13.8 & 15.2 & 10.0 \\
\hline Anode efficiency $\eta_{a}$ & 0.40 & 0.39 & 0.47 & 0.21 \\
\hline Mass utilization efficiency $\eta_{m}$ & 0.76 & $0.81^{\mathrm{a}}$ & 0.78 & $0.75^{\mathrm{a}}$ \\
\hline Beam current efficiency $\eta_{b}$ & 0.71 & 0.64 & 0.77 & 0.63 \\
\hline Beam voltage efficiency $\eta_{V}$ & 0.83 & $0.83^{\mathrm{b}}$ & 0.90 & $0.76^{\mathrm{b}}$ \\
\hline Beam divergence efficiency $\eta_{d}$ & 0.86 & N/A & 0.79 & N/A \\
\hline Charge efficiency $\eta_{c}$ & 0.99 & N/A & 0.99 & N/A \\
\hline $\mathrm{Xe}^{+}$current fraction & 0.90 & N/A & 0.84 & N/A \\
\hline $\mathrm{Xe}^{2+}$ current fraction & 0.10 & N/A & 0.16 & N/A \\
\hline
\end{tabular}




\section{List of captions}

Figure 1: Comparison between standard and magnetically shielded Hall thruster configurations.

Figure 2: Normalized magnetic field strength along the thruster centerline.

Figure 3: Computational meshes ( $75 \times 100$ grid cells). The stream function delimiting the four regions used for the electron fluid model and the cathode line are also shown.

Figure 4: Profiles of (a) ion velocity, (b) collision frequencies along the thruster centerline for the US configuration. The xenon mass flow is $1 \mathrm{mg} . \mathrm{s}^{-1}$ and the voltage $300 \mathrm{~V}$.

Figure 5: Profiles of (a) ion velocity, (b) collision frequencies along the thruster centerline for the MS configuration. The xenon mass flow is $1 \mathrm{mg} \cdot \mathrm{s}^{-1}$ and the voltage $300 \mathrm{~V}$.

Figure 6: Time-averaged plasma properties of the US configuration. (a) Contours of electric potential (from 0 to $300 \mathrm{~V}, 10$ contours equally spaced) and magnetic field lines, (b) 2D profile of electron temperature (color scale, 8 contours equally spaced, maximum of $30 \mathrm{eV}$ ), (c) $2 \mathrm{D}$ profile of ionization source term (color scale, 8 contours equally spaced, maximum of $9.2 \times 10^{23} \mathrm{~m}^{-3} \mathrm{~s}^{-1}$ ) overlaid by ion flux vectors,. The xenon mass flow is $1 \mathrm{mg} \cdot \mathrm{s}^{-1}$ and the voltage $300 \mathrm{~V}$.

Figure 7: Time-averaged plasma properties of the MS configuration. (a) Contours of electric potential (from 0 to $300 \mathrm{~V}, 10$ contours equally spaced, contours of 305 and $309 \mathrm{~V}$ are also shown) and magnetic field lines, (b) 2D profile of electron temperature (color scale, 8 contours equally spaced, maximum of $53 \mathrm{eV}$ ), (c) 2D profile of ionization source term (color scale, 8 contours equally spaced, maximum of $1.3 \times 10^{24} \mathrm{~m}^{-3} \mathrm{~s}^{-1}$ ) overlaid by ion flux vectors. The xenon mass flow is $1 \mathrm{mg} . \mathrm{s}^{-1}$ and the voltage $300 \mathrm{~V}$. 
Figure 8: Time variation of the discharge current for the US and MS configurations. The xenon mass flow is $1 \mathrm{mg} . \mathrm{s}^{-1}$ and the voltage $300 \mathrm{~V}$.

Figure 9: axial profile of (a) ion kinetic energy impacting inner and outer walls, (b) eroded thickness estimation for 1000 hours of thruster operation for a threshold of sputtering of 30 $\mathrm{eV}$, and for the US and MS configurations. The xenon mass flow is $1 \mathrm{mg} . \mathrm{s}^{-1}$ and the voltage $300 \mathrm{~V}$. 


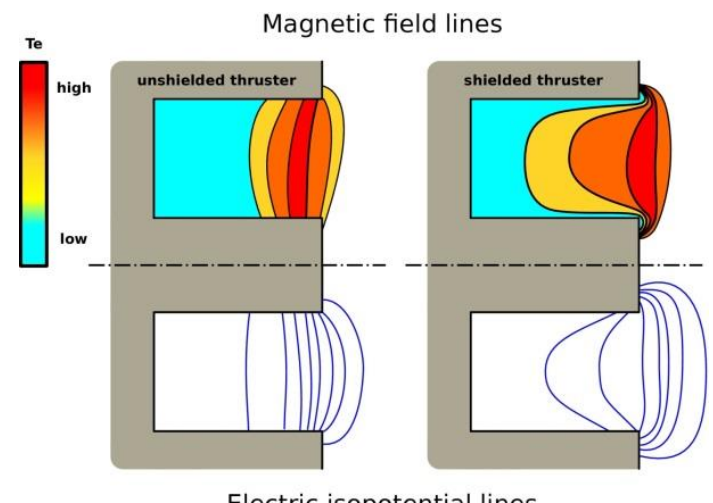

Figure 1. 


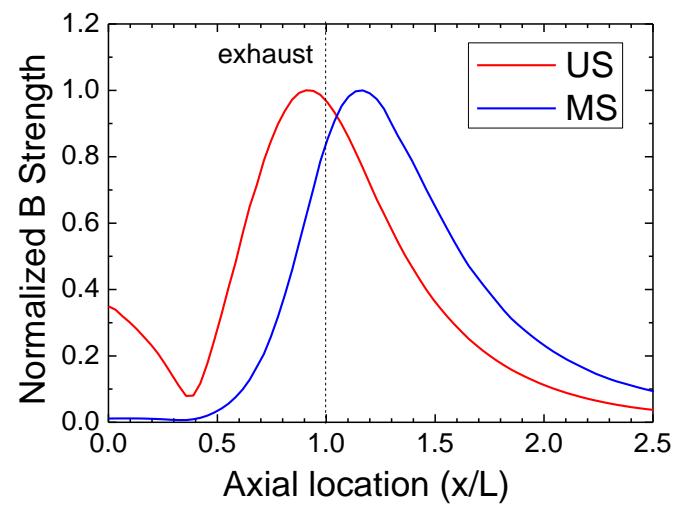

Figure 2. 


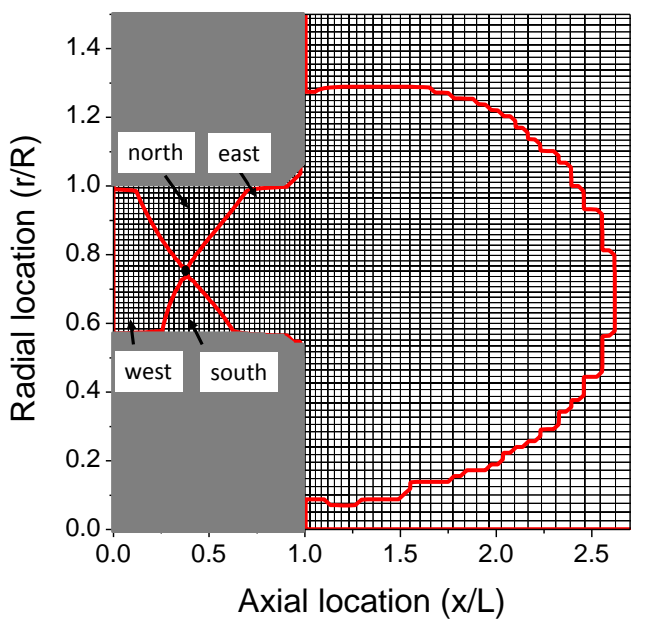

Figure 3. 

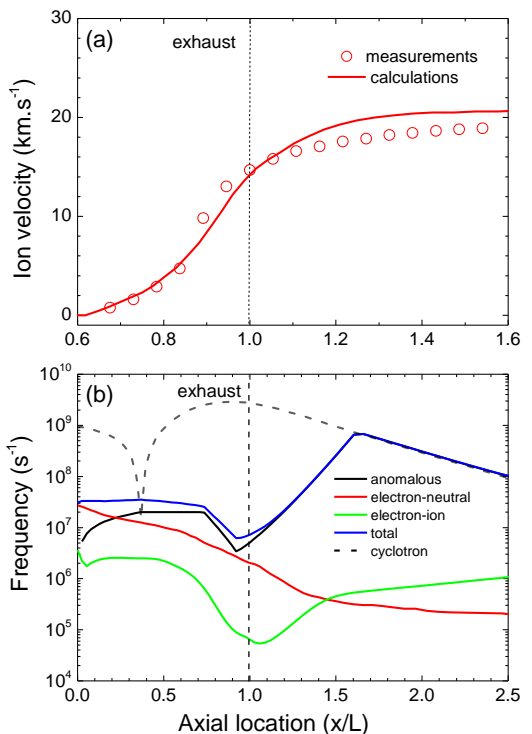

Figure 4. 

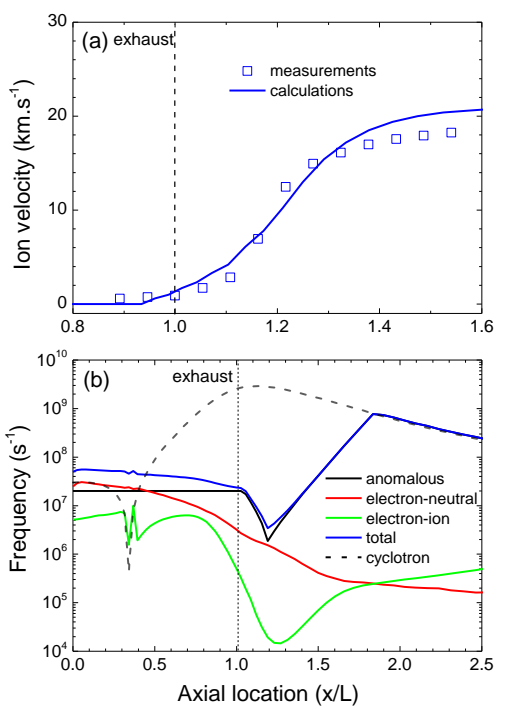

Figure 5. 

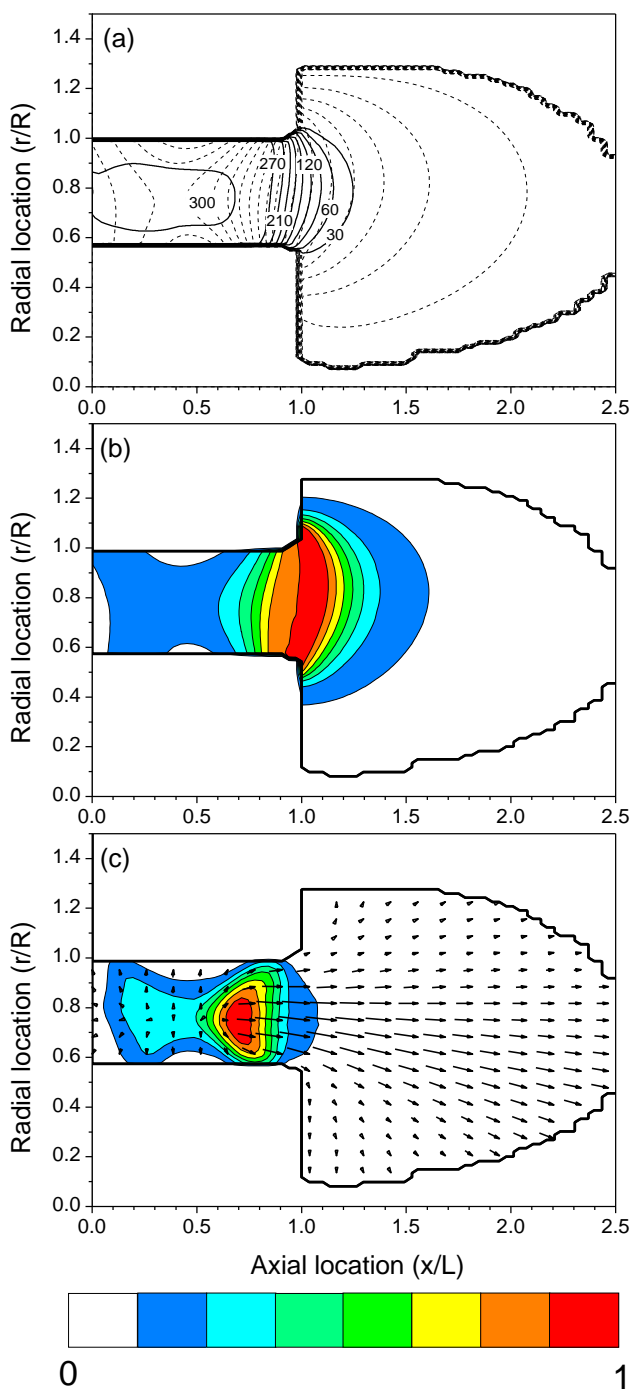

Figure 6. 


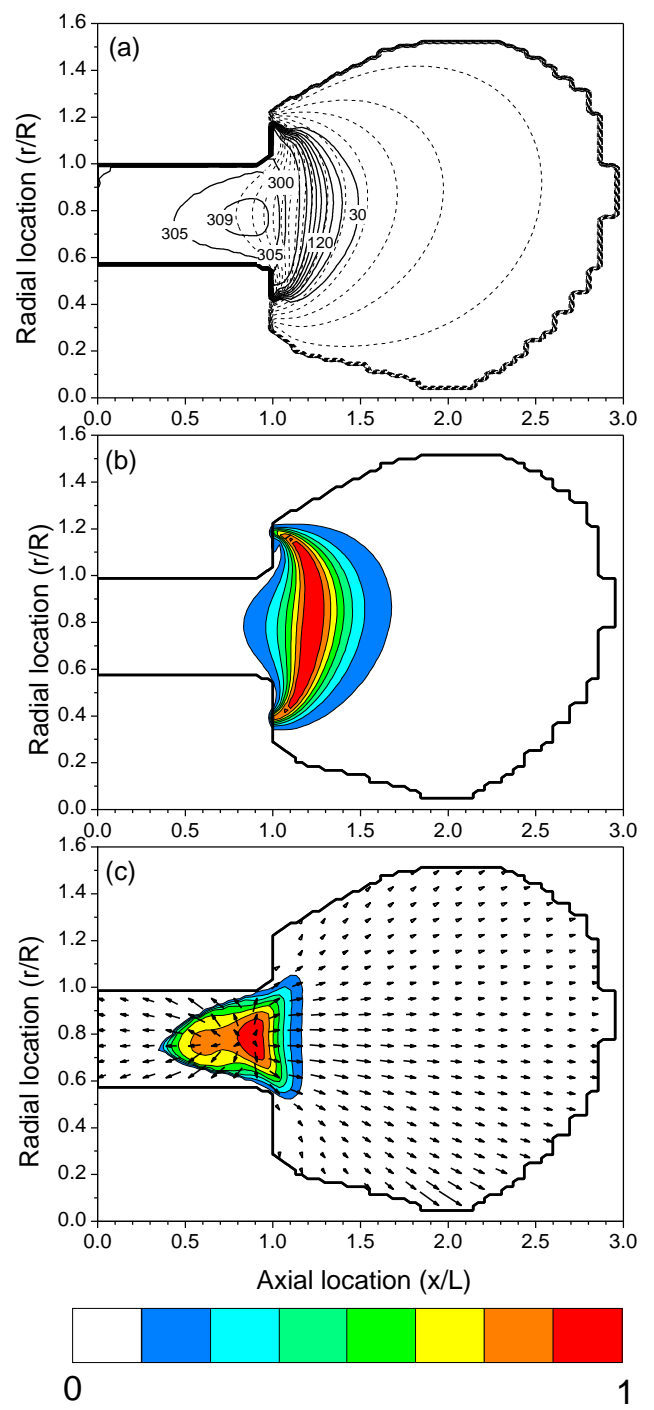

Figure 7. 


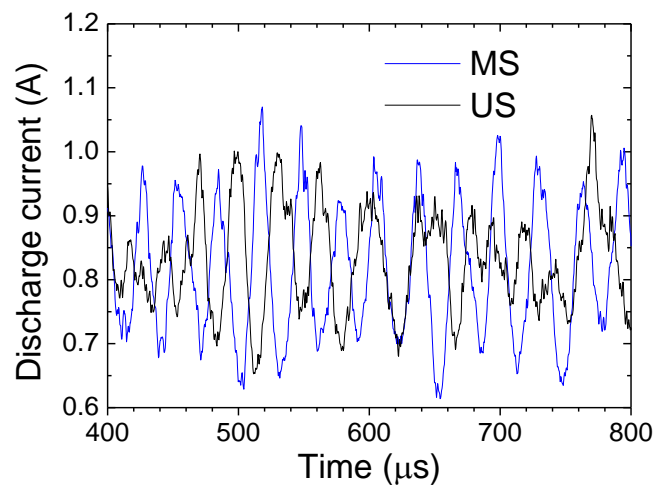

Figure 8. 

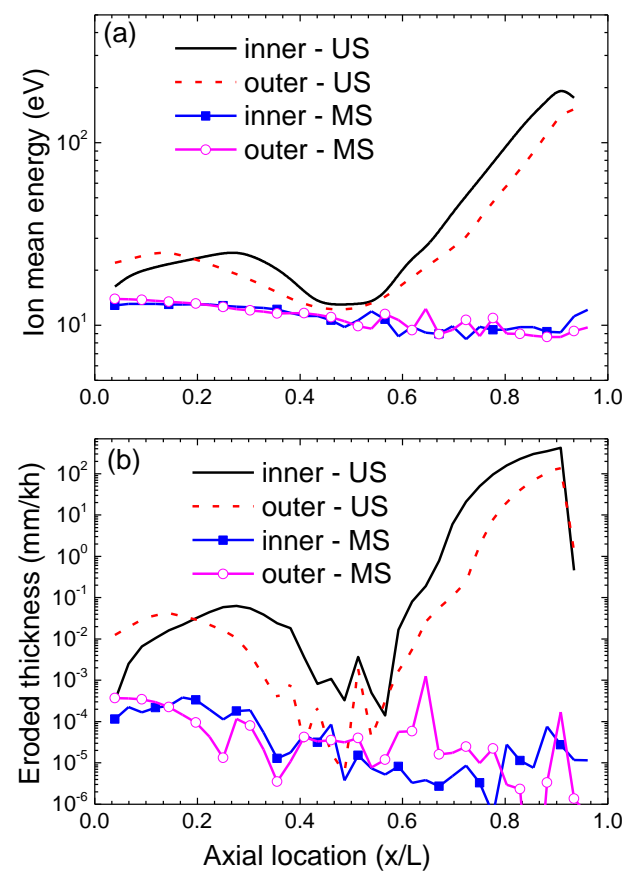

Figure 9. 